\title{
Acupuncture on the Stress-Related Drug Relapse to Seeking
}

\author{
Hyo Sun Roh, ${ }^{1}$ Bo Ra Park, ${ }^{2}$ Eun Young Jang, ${ }^{2}$ Jin Sook Kim $\mathbb{D}^{\circ},{ }^{3}$ and Young S. Gwak $\mathbb{D}^{2,4}$ \\ ${ }^{1}$ Department of Acupoint, College of Korean Medicine, Dongguk University, Gyeonggi-do 10326, Republic of Korea \\ ${ }^{2}$ Addiction Research Center and Department of Physiology, College of Korean Medicine, Daegu Haany University, \\ Daegu 42158, Republic of Korea \\ ${ }^{3}$ Addiction Research Center and Department of Secondary Special Education, Daegu Haany University, \\ Daegu 42158, Republic of Korea \\ ${ }^{4}$ Department of Anesthesiology, Wake Forest Baptist Medical Center, Winston Salem, NC 27106, USA
}

Correspondence should be addressed to Jin Sook Kim; jskim@dhu.ac.kr and Young S. Gwak; ygwak@wakehealth.edu

Received 11 November 2017; Revised 30 January 2018; Accepted 19 July 2018; Published 17 October 2018

Academic Editor: Mohammed S. Ali-Shtayeh

Copyright (c) 2018 Hyo Sun Roh et al. This is an open access article distributed under the Creative Commons Attribution License, which permits unrestricted use, distribution, and reproduction in any medium, provided the original work is properly cited.

\begin{abstract}
Drug addiction is a chronic relapsing disease, which causes serious social and economic problems. The most important trial for the successful treatment of drug addiction is to prevent the high rate of relapse to drug-seeking behaviors. Opponent process as a motivational theory with excessive drug seeking in the negative reinforcement of drug dependence reflects both loss of brain reward system and recruitment of brain stress system. The negative emotional state produced by brain stress system during drug withdrawal might contribute to the intense drug craving and drive drug-seeking behaviors via negative reinforcement mechanisms. Decrease in dopamine neurotransmission in the nucleus accumbens and recruitment of corticotropin-releasing factor in the extended amygdala are hypothesized to be implicated in mediating this motivated behavior. Also, a brain stress response system is hypothesized to increase drug craving and contribute to relapse to drug-seeking behavior during the preoccupation and anticipation stage of dependence caused by the exposure to stress characterized as the nonspecific responses to any demands on the body. Acupuncture has proven to be effective for reducing drug addiction and stress-related psychiatric disorders, such as anxiety and depression. Furthermore, acupuncture has been shown to correct reversible brain malfunctions by regulating drug addiction and stress-related neurotransmitters. Accordingly, it seems reasonable to propose that acupuncture attenuates relapse to drug-seeking behavior through inhibition of stress response. In this review, a brief description of stress in relapse to drug-seeking behavior and the effects of acupuncture were presented.
\end{abstract}

\section{Introduction}

Acupuncture, as a complementary and alternative therapeutic intervention, has been widely practiced as a therapeutic intervention in eastern countries for thousands of years. Various demonstration of acupuncture including manual, electrical, and laser on acupuncture points have suggested the therapeutic effect of acupuncture on mental and physical disorders [1-3]. Studies in animals and humans have demonstrated that acupuncture caused neurobiological responses in the brain by stimulating specific acupoints on the skin and muscle with insertion of fine acupuncture needles [4].

Acupuncture has been shown to be effective for reducing drug addiction in preclinical and clinical trials [5]. In particular, acupuncture has been accepted to be an effective detoxification method for drug addiction as a standard procedure [6]. Drug addiction is a chronically relapsing disease characterized by uncontrolled or compulsive use of addictive drugs that resulted in serious personal, social, and economic problems $[7,8]$. One of the main factors that cause relapses in addicts is the exposure to stress. Stress can be characterized as the nonspecific responses of the body to any demands for a change [9]. Indeed, previous studies suggested that stressrelated psychiatric disorders, such as anxiety and depression, commonly led to either drug relapse or the vulnerability to drug abuse [10-12]. Also, addicts displayed stress-induced negative emotions such as fear, sadness, and anger [13]. This suggests that psychological treatment would be an effective therapeutic strategy on the addiction treatment. 
Previous studies have suggested that a motivated behavior associated with excessive drug seeking in the negative reinforcement of drug dependence reflects both loss of brain reward function and recruitment of brain stress system. The negative emotional state produced by brain stress system during drug withdrawal might contribute to the intense drug craving and drive drug-seeking behaviors via negative reinforcement mechanisms [14]. Previous studies have shown that acupuncture plays an important role in correcting dysregulation of stress-related brain emotional systems and improves the efficiency of homeostatic mechanisms of emotion $[15,16]$. Also, acupuncture has been proven to be an effective treatment for reducing stress, anxiety, and depression via modulation of brain stress and endocrine systems in preclinical and clinical studies $[17,18]$. Furthermore, it has been suggested that acupuncture may be an adjunct therapy for the treatment of several mental disorders including drug addiction [19]. Thus, it is not unexpected that acupuncture ameliorates negative emotional states of the addiction cycle by regulating neurotransmitters in the brain that have long been associated with addictive behaviors [20]. Indeed, neurochemical studies have shown that acupuncture reduced not only anxiety-like behavior by normalizing catecholamine levels in the amygdala but also behavioral withdrawal syndromes by normalizing dopamine release in the nucleus accumbens (NAc) in ethanol-dependent rat [21]. Although many methods have been employed in reducing drug addiction, there is currently no satisfactory medical intervention to prevent relapse to drug seeking and there are still many unanswered questions about the basic mechanisms underlying the effectiveness of acupuncture in the treatment of drug relapse, including stress-induced reinstatement. In the present review, we focused on acupuncture's effectiveness in suppressing relapse to drug-seeking behavior by modulating brain stress response systems.

\section{Neuronal Adaptation on Drug Addiction}

Drug addiction has been suggested as a disorder showing impulsivity and compulsivity to seek and take the drug [22]. Previous studies have suggested that opponent process as a motivational theory with excessive drug seeking in the negative reinforcement of drug dependence reflects both loss of brain reward function and recruitment of brain stress system. The negative emotional state produced by brain stress system during drug withdrawal might contribute to the intense drug craving and drive drug-seeking behaviors via negative reinforcement mechanisms [14]. Since the exhilarating rush that reinforces the desire to take drugs occurs from impulsivity to compulsivity, positive reinforcement moves to negative reinforcement in driving the motivated behavior. Drug seeking is triggered by addictive drugs through proponent processes mediated by brain reward system, especially mesolimbic dopamine system, which provides positive reinforcement. The continued drug abuse induced by positive reinforcement causes a new neuroadaptive mechanism that resulted in the dysregulation of the brain reward pathways [22]. This counteradaptive process including the opponent process negatively affects the maintenance or recovery of homeostasis in the brain reward system. Based on an association between drug-taking behaviors and brain motivation, acute effect of a drug itself (positive hedonic effect) was hypothesized to be counteracted by a negative feedback control which led to decreased reward functions via altered set point of reward [23]. Therefore, the counteradaptive process increases drug craving and vulnerability to drug relapse to remove negative emotional state in dependence. This process was hypothesized to produce an altered allostatic state, a predominant neuroadaptive process in drug addiction.

The concept of allostasis was introduced to differentiate the chronic deviation of the homeostasis regulatory system from the normal condition to the newly established set point $[24,25]$. This set point was mainly characterized by activation of the hypothalamic-pituitary-adrenal (HPA) axis, the hallmark of the stress reaction [26], and the extrahypothalamic corticotropin-releasing factor (CRF) system of the extended amygdala, a primitive structure of brain stress response systems [27]. Activation of these systems is believed to be responsible for the mechanism, at least in part, underlying negative emotional state including anxiety and depression that accompanies drug withdrawal. Also, this activation contributes to the opponent process as a motivational theory causing relapses to drug-seeking behaviors in the negative reinforcement of drug dependence [14]. Hypofunctional state of reward systems in the opponent process reflects anxiety and anhedonia which accompanies drug withdrawal. These negative motivational states may contribute to vulnerability to relapse during the development of drug addiction [28].

\section{Effect of CRF and Stress on Drug Relapse}

Although the exact neurochemical mechanism mediating stress-induced relapse to drug seeking is not clearly understood, some evidence exists to suggest that stress has been implicated in drug reinstatement. Regarding relapse to drug seeking, mainly two ways exist in chronically sensitizing negative emotional states during acute withdrawal from chronic administration of abused drugs. One way is to develop protracted abstinence and the other way is to increase sensitivity to reinstatement of drug-seeking behavior, as indicated in stress-induced reinstatement [29]. The brain stress hormone, CRF, may be the motivational aspects of drug withdrawal such as ethanol withdrawal. The activation of CRF system in the extended amygdala during acute withdrawal from chronic drug administration may represent the mechanism underlying the opponent process in negative emotional states [30]. Indeed, previous studies have suggested that the brain CRF system plays an important role in mediating anxiety-like behavior and ethanol-seeking behavior in ethanol-dependent rats [31-34].

CRF neurons projecting from the central nucleus of the amygdala to the bed nucleus of the stria terminal mediate the stress-induced relapse to cocaine seeking [35]. Further support for a role of CRF is the significant overlap between the neural circuitry of stress-induced reinstatement and that of acute motivational withdrawal [36, 37]. Literature also demonstrated that the cortical glutamatergic activation contributed to the drug seeking and stress. It is well known that 
glutamatergic neurotransmission between the PFC and VTA regulate dopaminergic neuronal activity. For example, stress induced the increase of dopamine release in the PFC and NAc, which was attenuated by glutamate receptor inhibition in the VTA $[38,39]$. Activation of cortical glutamate neurons has also been implicated in stress-induced relapse to drugseeking. CRF release in the VTA induced by stress reinstated cocaine seeking through activation of glutamatergic neurons in the VTA and glutamatergic activation in the NAc projecting from prefrontal cortex $[40,41]$. Stress also has a significant impact on the mesolimbic dopamine system. Stress reinstated drug-seeking behavior by increasing dopamine release in the NAc, a primitive structure that is responsible for the reinforcing effect of drugs [42], which was blocked by local injection of dopamine receptor antagonist into the NAc [43].

Although not much is known about the neural circuits between the CRF and mesolimbic dopamine system, significant evidence supports an interaction between CRF and dopamine systems in the extended amygdala and the VTA leading to the development of drug dependence and relapse. Drug-withdrawal results in a sustained activation of the CRF system in the extended amygdala. Consequently, the brain CRF system causes the reduction of accumbal dopamine release in drug-dependent and withdrawn subjects [44]. More specifically, chronic hyperactivity of the CRF system produces transient dysfunction of dopaminergic neurons in the brain through oxidative mechanisms. On the other hand, acute stress increases dopamine release in the NAc shell through activation of CRF pathway [45]. In support of this observation, a recent study has shown that exposure to external stressors exacerbates CRF release in the VTA during drug withdrawal in dependent subjects and arouses drug relapse through activation of dopaminergic neurons [46].

\section{Effect of Acupuncture on Relapse in Drug Dependence}

Although few experiments have investigated acupunctural effect on drug relapse, results of animal and clinical studies provided evidence for acupuncture-induced inhibition of drug reward and stress systems in drug dependence. Since the reduction of dopamine levels in the NAc during withdrawal from ethanol administration may be responsible for the negative affect such as anxiety and anhedonia [47, 48], it seems reasonable to propose that acupuncture may reduce the motivated behavior in alcohol dependence by restoring the deficiency in dopamine release in the brain. Previous works have demonstrated that acupuncture at HT7 effectively prevented both the decrease of extracellular dopamine levels in the NAc during ethanol withdrawal and the increase in accumbal dopamine levels induced by the ethanol challenge [49]. In addition, electroacupuncture (EA) attenuated alcohol drinking behavior by enhancing dopamine levels in immobilized rats [50]. Inhibitions of anxiety-like behavior and reductions in tyrosine hydroxylase mRNA expression in the VTA and tyrosine hydroxylase protein expression in the NAc by acupuncture in ethanol-withdrawn rats confirmed these results [51]. Furthermore, the anxiolytic effect of acupuncture was blocked by local infusion of dopamine receptor antagonists SCH23390 (the selective D1 receptor antagonist) and eticlopride (the selective D2 receptor antagonist) into the NAc [52]. Taken together, these findings provide evidence that acupuncture may help to suppress drug relapse and craving by inhibiting dopamine depletion in the mesolimbic dopamine system.

4.1. Effect of Acupuncture on BDNF. One study attempted to determine a role for BDNF in the anxiolytic effects of acupuncture. This study showed that acupuncture inhibited anxiety-like behaviors by ameliorating the dysfunction of the mesolimbic dopamine pathway through inhibition of BDNF expression in the VTA in ethanol-dependent rats [51]. However, a role for BDNF in the motivated behavior and the mesolimbic dopamine system in drug dependence still remains unclear. Results of animal studies showed that withdrawal from chronic opiates increased BDNF levels and BDNF-related neuronal plasticity in the VTA and produced aversive withdrawal motivation [53]. Similarly, specific knockdown of viral-mediated BDNF in the mesolimbic dopamine pathway ameliorated social aversion [54, 55]. BDNF levels in the NAc significantly increased in chronic methamphetamine-induced depression-like behavior [56]. Importantly, BDNF has been shown to serve as a key regulator of the mesolimbic dopamine system $[57,58]$. Thus, it can be expected that acupuncture can reduce drug relapse by improving negative emotional state in drug dependence through modulation of the BDNF-mediated mesolimbic dopamine pathway. In contrast, it has been shown that repeated electroacupuncture at Baihui (GV-20) and Yintang (EX-HN3) increased glutamate, 5-hydroxytryptamine (5$\mathrm{HT}$ ), and the BDNF-related peptides, such as protein kinase $\mathrm{C}$ (PKC) and cyclic adenosine monophosphate response element binding protein, in the hippocampus and inhibited stress-induced depression [59]. Although few clinical studies have investigated the effect of acupuncture on brain neurotransmitter levels, it has been shown that acupuncture reduced serum norepinephrine levels and increased serum levels of glutamate, 5-HT, and BDNF in insomnia patients, suggesting that serum BDNF has been implicated in the sedative effect of acupuncture $[60,61]$.

4.2. Effect of Acupuncture on Cannabinoid. Cannabinoid 1 receptor (CB1R) has been suggested to mediate ethanol self-administration by acting on the mesolimbic dopamine pathway [62]. Interestingly, electroacupuncture (EA) attenuated upregulation of CB1R in the striatum, amygdala, and VTA during ethanol withdrawal [63]. Activation of CB1R expressed on VTA GABA neurons inhibited GABAergic transmission and in turn increased dopamine transmission in the NAc [64]. Therefore, it is reasonable to propose that CB1R-mediated inhibition of GABA neurons in the VTA by EA may inhibit hypoexcitability of dopamine neurons in the mesolimbic system during withdrawal from chronic ethanol administration. In addition, cannabinoid agonist HU210 promoted relapse and cocaine-seeking behaviors attenuated by the selective CB1 receptor antagonist SR141716A and inhibition of CB1R attenuated ethanol self-administration 
and alcohol-seeking behavior [65, 66]. These data revealed an important role of the cannabinoid system in the neuronal processes underlying relapse to cocaine and alcohol seeking and provided a rationale for the use of cannabinoid receptor antagonists for the prevention of relapse to cocaine and alcohol use. As alcoholics expressed CB1R polymorphism and contributed to ethanol dependence [67], EA may reduce alcohol-seeking behavior by modulating CB1R in ethanoldependent subjects.

4.3. Effect of Acupuncture on Opioid. Over a decade, literatures have shown that acupuncture reduced alcohol cravings in alcohol-dependent patients. The microdialysis study raised the possibility that the GABA pathway may mediate acupuncture inhibition of withdrawal syndrome and reduction of dopamine release in the NAc in ethanol-withdrawn rats [49]. In addition, acupuncture decreased ethanol selfadministration and suppressed VTA GABA neuronal firing rate through activation of endogenous opioid systems [68]. Previous works have stated that acupuncture may activate $\beta$-endorphinergic and enkephalinergic neurons in the arcuate nucleus of the hypothalamus [69, 70]. For example, electroacupuncture suppressed morphine withdrawal with a significant increase of brain beta-endorphin level [71]. $\beta$ Endorphinergic neurons projecting from the arcuate nucleus may trigger opioid receptors on GABA neurons in the VTA and NAc [72]. It is well known that GABA interneurons and GABAergic terminal projected from the NAc synapse with dopamine neurons in the VTA. Therefore, beta-endorphin and endogenous mu-opioid peptides may disinhibit GABA neuronal activity in the NAc and/or VTA that would result in the enhancement of dopamine neuron activity in the VTA, thereby increasing dopamine release in the NAc. Thus, it can be hypothesized that acupuncture may inhibit drugseeking behavior and craving by normalizing the depletion of dopamine in the NAc via activation of $\beta$-endorphinergic neurons from the arcuate nucleus of the hypothalamus [73]. Furthermore, it has been suggested that $\beta$-endorphin in the brain modulated behavioral responses to stress and anxiety in drug-seeking behavior [74]. Although the exact neurochemical mechanism regarding a role for $\beta$-endorphin is still elusive, some evidence suggests that $\beta$-endorphin may reduce stress response through inhibition of CRH release [75]. In support of this evidence, pharmacological studies have shown that low frequency EA $(2 \mathrm{~Hz})$ inhibited morphine-induced conditioned preference place by increasing enkephalin synthesis in the NAc [76].

4.4. Effect of Acupuncture on Stress Response. It is well documented that acupuncture decreases anxiety levels and stressrelated symptoms [77, 78]. In addition, studies have demonstrated that acupuncture reduces stress response by the following mechanisms: (1) inhibition of the sympathoadrenal medullary system in rats exposed to immobilization stress [79], (2) inhibition of HPA system in maternal separation rats [80], and (3) suppression of HPA system in ethanolwithdrawn rats $[80,81]$, respectively.

Many evidences have suggested that the brain stress response system may be an important factor in drug-seeking behavior of drug dependence. Amygdala is a center for the modulation of affective behaviors, such as anxiety, depression, and stress that trigger drug seeking and relapse in drug addiction. Many studies reported that chronic treatment of ethanol, nicotine, or cannabinoid commonly increased CRF levels in the central nucleus of the amygdala whereas CRF antagonists in the amygdala reduced anxiety-like behavior [82-84]. Also, local infusion of CRF receptor antagonist into the central nucleus of the amygdala was reported to inhibit ethanol-seeking behavior in ethanol-withdrawn rats [85]. These suggest that the amygdala may play an important role in mediating stress-induced reinstatement of drug-seeking and anxiety-like response presumably through activation of CRF neurons input to the amygdala. In addition, electroacupuncture attenuated morphine withdrawal and c-Fos expression in the central nucleus of the amygdala and stressinduced CRH level rats [86, 87]. Interestingly, one study has shown that acupuncture inhibited anxiety-like behavior and increases in plasma corticosterone and amygdaloid catecholamine in ethanol-withdrawn rats [85]. Based on CRFnoradrenergic interaction in the amygdala, it was suggested that acupuncture may reduce ethanol withdrawal dysphoric symptoms by modulating the stress response system. Moreover, CRF in the central nucleus of the amygdala has been implicated in the anxiolytic effect of acupuncture in ethanol-withdrawn rat [88]. CRF in the amygdala has been shown to be important in mediating anxiety-like behavior that accompanies ethanol withdrawal and contributed to relapse to ethanol-seeking behavior and drug craving [88]. Therefore, these results raise the possibility that acupuncture may attenuate anxiety-like behavior during ethanol withdrawal through suppression of CRF neurons in the amygdala. Consistent with this finding, acupuncture reduced anxietylike behavior during nicotine withdrawal by inhibiting CRF expression in the amygdala [89]. These effects have been confirmed in a variety of experimental studies showing that acupuncture suppressed CRF neuronal activity in the various regions of brain including the amygdala [90, 91]. Interestingly, $\beta$-endorphin is important in reducing response of the hypothalamic CRF neuron to stress and attenuating anxiety-like behavior $[92,93]$. Thus, acupuncture inhibition of drug-seeking behavior might result from modulation of stress response system in the amygdala through activation of $\beta$-endorphinergic neurons in the arcuate nucleus of the hypothalamus.

4.5. Acupuncture on Stress-Induced Relapse to Drug Seeking. Multiple types of stimuli trigger craving and subsequent relapse in drug addicts, such as exposure to the drug itself, stress, and drug-associated cues [94-96]. As these stimuli activate dopamine neurons in the VTA followed by increase dopamine release in the NAc, the mesolimbic dopamine system plays an important role in relapse to drugseeking behavior $[97,98]$. More specifically, drug dependents subjected to external stressors revealed increases in drug craving and relapse. This drug relapse induced by stress was suggested to be motivated by self-medication of the associated negative effect of stress [99] and CRF played a key role in mediating stress-induced drug reinstatement [100]. 
Acupuncture-initiated impulses may activate brain pathways and thus ameliorate the efficiency of homeostatic mechanisms of stress and reward mechanism in the brain [101,102]. Previous work using cocaine self-administration has shown that acupuncture attenuated stress-induced relapse to cocaine seeking by reducing neuronal activity in the NAc [103]. As acute stress enhances dopamine release in the NAc via activation of CRF neurons [45], this study provided evidence that acupuncture might attenuate relapse to drug seeking by the reduced dopamine response via activation of GABA neurons in the VTA. Theoretically, VTA GABA neurons inhibit the activity of dopamine neurons in the VTA and critically modulate drug reward and relapse [104]. Indeed, activation of VTA GABA neurons by optogenetic stimulation inhibits dopamine neuronal activity in the VTA and in turn decreases dopamine release in the NAc [105]. Interestingly, the recent study showed that acupuncture at Shenmen (HT7) points attenuated cocaine-primed reinstatement, cocaine inhibition of GABA neuron activity in the VTA, and acute cocaine-induced dopamine release in the NAc, which were reversed by local infusion of the selective GABAB receptor antagonist 2-hydroxysaclofen [106]. These results suggest that acupuncture may reduce relapse to cocaine-seeking behavior by enhancement of GABAergic inhibition in the VTA.

Considerable studies have investigated the effect of acupuncture on the mesolimbic dopamine system and relapse to drug-seeking behavior induced by drug, drug-associated cues, and stress. Literature demonstrated that EA reduced cue-evoked heroin-seeking and reinstatement of heroinseeking by heroin priming and extinction responding of heroin-seeking behavior and Fos expression in the NAc core $[105,107]$. In addition, the functional MRI (fMRI) study has shown that acupuncture inhibited cue-induced heroin craving and brain activation [108]. These results suggest the possibility that acupuncture may reduce discrete cueinduced drug-seeking behavior by modulating dopamine release in the NAc. Also, it has been shown that acupuncture reduced morphine or cue-induced reinstatement of morphine-seeking behaviors and morphine craving in progressive ratio via activation of GABA receptors $[109,110]$. Similarly, acupuncture decreased extracellular dopamine levels in the NAc and behavioral sensitization induced by repeated morphine administration [111]. Given that drug sensitization may induce relapse to abused drugs, it can be suggested that acupuncture may reduce morphine-seeking behavior. This suggestion is supported by the finding that acupuncture inhibited behavioral sensitization induced by repeated cocaine administration via modulation of dopamine neuron activity in the VTA [112].

\section{Acupuncture in Clinical Therapy}

Over a decade, the clinical applications of acupuncture to the prevention or attenuation of drug craving or relapse have been suggested $[113,114]$. Clinical studies have shown that chronic acupuncture [twice a week for weeks, Zhubin (KI9) acupoint)] reduced alcohol craving in alcoholdependent patients using measures of the Visual Analogue Scale (VAS) [18] and transcutaneous electrical acupoint stimulation (TEAS, for 3 months) decreased the relapse rate of rehabilitating heroin user [115]. Acupuncture attenuated both ethanol intake and relapse in alcoholics [116, 117]. Acupuncture also has proven to be an effective treatment for reducing stress, anxiety, and depression via modulation of brain stress and endocrine systems in clinical studies [118]. The fMRI studies reported that acupuncture increased the self-alcohol abstinence in social alcohol drinkers and activated brain areas that have been associated with selfcontrol over alcohol craving [119] and reduced craving by smoking-related visual cues [120]. Other studies have reported that electroacupuncture suppressed the relapse rate and plasma $\beta$-endorphin and dynorphin A levels in heroin addicts and the prolonged acupuncture treatment showed dominant control effects on heroin craving behaviors with little side effects [121, 122]. Importantly, acupuncture's role has been reviewed in treating drug dependence in drugdependent patients including opioid, nicotine, cocaine, and alcohol [123]. Interestingly, the recent fMRI studies have suggested that acupuncture improved depressive behaviors in depressive patients through modulation of limbic system especially amygdala and subgenual anterior cingulate cortex [124] and the corticostriatal reward circuitry [125]. Based on an association between depression and drug relapse in dependence, these data raise the possibility for acupuncture's role in suppressing drug relapse. Taken together, these clinical studies provide evidence that acupuncture may be an effective therapy for reducing drug relapse to drug-seeking behaviors.

\section{Summary}

There is a lot of interest in gaining a better understanding of how acupuncture works in the brain related to addictive behaviors. There are numerous studies in laboratory animals and humans in the field of acupuncture and drug addiction, and such researches have contributed to the significant improvement in determining the basic mechanisms of acupuncture in the treatment of drug addiction. However, the fact that a significant stress gives an excuse for the compulsive drug use and loss of control over drug is an important factor to elucidate the biological mechanism of drug addiction. This stress-induced psychological positive anticipation triggers and facilitates relapse to drug-seeking behaviors. Also, dysregulation of brain reward mechanisms is believed to involve the mesolimbic dopamine system and the brain stress system in some way.

In conclusion, studies provide strong evidences that acupuncture reduces stress-induced negative emotional state and relapse to drug-seeking behavior by modulating the mesolimbic dopamine system and CRF stress system. A better understanding of acupuncture's role may lead to the development of successful therapeutic intervention in the treatment of drug addiction associated with stress.

\section{Conflicts of Interest}

The authors declare no conflicts of interests exist. 


\section{Acknowledgments}

This work was supported by the National Research Foundation of Korea (NRF) grants funded by the Korean Government (MSIP) (NRF-2015R1A5A7037508, NRF2014R1A1A4A01004179, and NRF-2014R1A2A2A01005851).

\section{References}

[1] Y.-C. Yun, D. Jang, S.-B. Yoon, D. Kim et al., "Laser Acupuncture Exerts Neuroprotective Effects via Regulation of Creb, Bdnf, Bcl2, and Bax Gene Expressions in the Hippocampus," EvidenceBased Complementary and Alternative Medicine, vol. 2017, Article ID 7181637, 11 pages, 2017.

[2] K. Takagi, N. Tanahashi, N. Amagasu et al., "Effect of Manual Acupuncture Stimulation at "Bai-Hui" (GV 20) or "Yintáng" (Ex-HN3) on Depressed Rats," Journal of Acupuncture and Meridian Studies, vol. 10, no. 1, pp. 26-32, 2017.

[3] T.-J. Ho, C.-W. Lee, Z.-Y. Lu et al., "Effects of Electroacupuncture on Methamphetamine-Induced Behavioral Changes in Mice," Evidence-Based Complementary and Alternative Medicine, vol. 2017, Article ID 5642708, 11 pages, 2017.

[4] "NIH Consensus Conference. Acupuncture," JAMA, vol. 280, no. 17, pp. 1518-1524, 1998.

[5] F. E. Motlagh, F. Ibrahim, R. A. Rashid, T. Seghatoleslam, and $\mathrm{H}$. Habil, "Acupuncture therapy for drug addiction," Chinese Medicine, vol. 11, article 16, 2016.

[6] M. Shwartz, R. Saitz, K. Mulvey, and P. Brannigan, "The value of acupuncture detoxification programs in a substance abuse treatment system," Journal of Substance Abuse Treatment, vol. 17, no. 4, pp. 305-312, 1999.

[7] D. I. Lubman, M. Yücel, and C. Pantelis, "Addiction, a condition of compulsive behaviour? Neuroimaging and neuropsychological evidence of inhibitory dysregulation," Addiction, vol. 99, no. 12, pp. 1491-1502, 2004.

[8] A. T. McLellan, D. C. Lewis, C. P. O’Brien, and H. D. Kleber, "Drug dependence, a chronic medical illness implications for treatment, insurance, and outcomes evaluation," Journal of the American Medical Association, vol. 284, no. 13, pp. 1689-1695, 2000.

[9] H. Selye, The Stress of Life, McGraw-Hill, New York, NY, USA, 1978.

[10] R. Sinha, "How does stress lead to risk of alcohol relapse?" Alcohol Research: Current Reviews, vol. 34, no. 4, pp. 432-440, 2012.

[11] J. R. McReynolds, E. M. Doncheck, Y. Li et al., "Stress Promotes Drug Seeking Through Glucocorticoid-Dependent Endocannabinoid Mobilization in the Prelimbic Cortex," Biological Psychiatry, vol. 84, no. 2, pp. 85-94, 2018.

[12] Z. L. Zhao, G. W. Zhao, H. Z. Li et al., "Acupuncture Attenuates Anxiety-Like Behavior by Normalizing Amygdaloid Catecholamines during Ethanol Withdrawal in Rats," EvidenceBased Complementary and Alternative Medicine, vol. 2011, Article ID 429843, 8 pages, 2011.

[13] G. F. Koob, "The role of CRF and CRF-related peptides in the dark side of addiction," Brain Research, vol. 1314, pp. 3-14, 2010.

[14] G. F. Koob and M. Le Moal, "Neurobiological mechanisms for opponent motivational processes in addiction," Philosophical Transactions of the Royal Society B: Biological Sciences, vol. 363, no. 1507, pp. 3113-3123, 2008.
[15] S. J. Russo and E. J. Nestler, "The brain reward circuitry in mood disorders," Nature Reviews Neuroscience, vol. 14, no. 9, pp. 609625, 2013.

[16] M. Pavuluri, K. Volpe, and A. Yuen, "Nucleus accumbens and its role in reward and emotional circuitry: A potential hot mess in substance use and emotional disorders," AIMS Neuroscience, vol. 4, no. 1, pp. 52-70, 2017.

[17] F. Carvalho, K. Weires, M. Ebling, M. De Souza Rabbo Padilha, Y. A. Ferrão, and R. Vercelino, "Effects of acupuncture on the symptoms of anxiety and depression caused by premenstrual dysphoric disorder," Acupuncture in Medicine, vol. 31, no. 4, pp. 358-363, 2013.

[18] J.-S. Lee, S.-G. Kim, T.-G. Jung, W. Y. Jung, and S.-Y. Kim, “Effect of Zhubin (KI9) acupuncture in reducing alcohol craving in patients with alcohol dependence: a randomized placebocontrolled trial," Chinese Journal of Integrative Medicine, vol. 21, no. 4, pp. 307-311, 2015.

[19] Y.-H. J. Kim, E. Schiff, J. Waalen, and M. Hovell, "Efficacy of acupuncture for treating cocaine addiction: A review paper," Journal of Addictive Diseases, vol. 24, no. 4, pp. 115-132, 2005.

[20] K. Pilkington, "Acupuncture therapy for psychiatric illness," International Review of Neurobiology, vol. 111, pp. 197-216, 2013.

[21] B. H. Lee, R. J. Zhao, J. Y. Moon et al., "Differential involvement of GABA system in mediating behavioral and neurochemical effect of acupuncture in ethanol-withdrawn rats," Neuroscience Letters, vol. 443, no. 3, pp. 213-217, 2008.

[22] G. F. Koob and M. Le Moal, "Drug addiction, dysregulation of reward, and allostasis," Neuropsychopharmacology, vol. 24, no. 2, pp. 97-129, 2001.

[23] G. F. Koob, "Alcoholism: Allostasis and beyond," Alcoholism: Clinical and Experimental Research, vol. 27, no. 2, pp. 232-243, 2003.

[24] B. S. McEwen, "Allostasis and allostatic load: implications for neuropsychopharmacology," Neuropsychopharmacology, vol. 22, no. 2, pp. 108-124, 2000.

[25] J. Schulkin, B. S. McEwen, and P. W. Gold, "Allostasis, amygdala, and anticipatory angst," Neuroscience \& Biobehavioral Reviews, vol. 18, no. 3, pp. 385-396, 1994.

[26] G. Koob and M. J. Kreek, "Stress, dysregulation of drug reward pathways, and the transition to drug dependence," The American Journal of Psychiatry, vol. 164, no. 8, pp. 1149-1159, 2007.

[27] O. George, M. Le Moal, and G. F. Koob, "Allostasis and addiction: Role of the dopamine and corticotropin-releasing factor systems," Physiology \& Behavior, vol. 106, no. 1, pp. 5864, 2012.

[28] G. F. Koob and M. Le Moal, "Addiction and the brain antireward system," Annual Review of Psychology, vol. 59, pp. 29-53, 2008.

[29] G. F. Koob, "Brain stress systems in the amygdala and addiction," Brain Research, vol. 1293, pp. 61-75, 2009.

[30] R. M. Richter and F. Weiss, "In vivo CRF release in rat amygdala is increased during cocaine withdrawal in self-administering rats," Synapse, vol. 32, no. 4, pp. 254-261, 1999.

[31] D. H. Overstreet, D. J. Knapp, and G. R. Breese, "Modulation of multiple ethanol withdrawal-induced anxiety-like behavior by CRF and CRF1 receptors," Pharmacology Biochemistry \& Behavior, vol. 77, no. 2, pp. 405-413, 2004.

[32] T. J. Phillips, C. Reed, and R. Pastor, "Preclinical evidence implicating corticotropin-releasing factor signaling in ethanol consumption and neuroadaptation," Genes, Brain and Behavior, vol. 14, no. 1, pp. 98-135, 2015. 
[33] K. I. Gabriel, C. L. Yu, J. A. Osborn, and J. Weinberg, "Prenatal ethanol exposure alters sensitivity to the effects of corticotropin-releasing factor (CRF) on behavior in the elevated plus-maze," Psychoneuroendocrinology, vol. 31, no. 9, pp. 10461056, 2006

[34] C. K. Funk, E. P. Zorrilla, M.-J. Lee, K. C. Rice, and G. F. Koob, "Corticotropin-Releasing Factor 1 Antagonists Selectively Reduce Ethanol Self-Administration in EthanolDependent Rats," Biological Psychiatry, vol. 61, no. 1, pp. 78-86, 2007.

[35] S. Erb, N. Salmaso, D. Rodaros, and J. Stewart, "A role for the CRF-containing pathway from central nucleus of the amygdala to bed nucleus of the stria terminalis in the stress-induced reinstatement of cocaine seeking in rats," Psychopharmacology, vol. 158, no. 4, pp. 360-365, 2001.

[36] L. Stinus, M. Le Moal, and G. F. Koob, "Nucleus accumbens and amygdala are possible substrates for the aversive stimulus effects of opiate withdrawal," Neuroscience, vol. 37, no. 3, pp. 767-773, 1990.

[37] R. Sinha, "The role of stress in addiction relapse," Current Psychiatry Reports, vol. 9, no. 5, pp. 388-395, 2007.

[38] P. Enrico, M. Bouma, J. B. de Vries, and B. H. C. Westerink, “The role of afferents to the ventral tegmental area in the handling stress-induced increase in the release of dopamine in the medial prefrontal cortex: a dual-probe microdialysis study in the rat brain," Brain Research, vol. 779, no. 1-2, pp. 205-213, 1998.

[39] P. W. Kalivas, P. Duffy, and J. Barrow, "Regulation of the mesocorticolimbic dopamine system by glutamic acid receptor subtypes," The Journal of Pharmacology and Experimental Therapeutics, vol. 251, no. 1, pp. 378-387, 1989.

[40] B. Wang, Y. Shaham, D. Zitzman, S. Azari, R. A. Wise, and Z.-B. You, "Cocaine experience establishes control of midbrain glutamate and dopamine by corticotropin-releasing factor: a role in stress-induced relapse to drug seeking," The Journal of Neuroscience, vol. 25, no. 22, pp. 5389-5396, 2005.

[41] K. McFarland, C. C. Lapish, and P. W. Kalivas, "Prefrontal glutamate release into the core of the nucleus accumbens mediates cocaine-induced reinstatement of drug-seeking behavior," The Journal of Neuroscience, vol. 23, no. 8, pp. 3531-3537, 2003.

[42] Y. Shaham, S. Erb, and J. Stewart, "Stress-induced relapse to heroin and cocaine seeking in rats: A review," Brain Research Reviews, vol. 33, no. 1, pp. 13-33, 2000.

[43] Z.-X. Xi, J. Gilbert, A. C. Campos et al., "Blockade of mesolimbic dopamine $\mathrm{D} 3$ receptors inhibits stress-induced reinstatement of cocaine-seeking in rats," Psychopharmacology, vol. 176, no. 1, pp. 57-65, 2004.

[44] E. Izzo, P. P. Sanna, and G. F. Koob, "Impairment of dopaminergic system function after chronic treatment with corticotropinreleasing factor," Pharmacology Biochemistry \& Behavior, vol. 81, no. 4, pp. 701-708, 2005.

[45] J. C. Lemos, M. J. Wanat, J. S. Smith et al., "Severe stress switches CRF action in the nucleus accumbens from appetitive to aversive," Nature, vol. 490, no. 7420, pp. 402-406, 2012.

[46] E. N. Holly, J. F. Debold, and K. A. Miczek, "Increased mesocorticolimbic dopamine during acute and repeated social defeat stress: Modulation by corticotropin releasing factor receptors in the ventral tegmental area," Psychopharmacology, vol. 232, no. 24, pp. 4469-4479, 2015.

[47] G. F. Koob, "A Role for Brain Stress Systems in Addiction," Neuron, vol. 59, no. 1, pp. 11-34, 2008.
[48] S. Lammel, B. K. Lim, and R. C. Malenka, "Reward and aversion in a heterogeneous midbrain dopamine system," Neuropharmacology, vol. 76, pp. 351-359, 2014.

[49] R. J. Zhao, S. S. Yoon, B. H. Lee et al., "Acupuncture normalizes the release of accumbal dopamine during the withdrawal period and after the ethanol challenge in chronic ethanol-treated rats," Neuroscience Letters, vol. 395, no. 1, pp. 28-32, 2006.

[50] K. Yoshimoto, B. Kato, K. Sakai, M. Shibata, T. Yano, and M. Yasuhara, "Electroacupuncture stimulation suppresses the increase in alcohol-drinking behavior in restricted rats," Alcoholism: Clinical and Experimental Research, vol. 25, 6, pp. 63S68S, 2001.

[51] Z. Zhao, S. C. Kim, R. Zhao et al., "The tegmental-accumbal dopaminergic system mediates the anxiolytic effect of acupuncture during ethanol withdrawal," Neuroscience Letters, vol. 597, pp. 143-148, 2015.

[52] W. Xia, N.-N. Chu, J. Liang et al., "Electroacupuncture of $2 \mathrm{~Hz}$ Has a Rewarding Effect: Evidence from a Conditioned Place Preference Study in Rats," Evidence-Based Complementary and Alternative Medicine, vol. 2011, Article ID 730514, 6 pages, 2011.

[53] H. Vargas-Perez, A. Bahi, M. R. Bufalino et al., "BDNF signaling in the VTA links the drug-dependent state to drug withdrawal aversions," The Journal of Neuroscience, vol. 34, no. 23, pp. 78997909, 2014.

[54] O. Berton, C. A. McClung, R. J. DiLeone et al., "Essential role of BDNF in the mesolimbic dopamine pathway in social defeat stress," Science, vol. 311, no. 5762, pp. 864-868, 2006.

[55] J. Wang, R. M. Bastle, and E. M. Nikulina, "VTA BDNF enhances social stress-induced compulsive cocaine bingeing," Oncotarget, vol. 8, no. 4, pp. 5668-5669, 2017.

[56] Q. Ren, M. Ma, C. Yang, J.-C. Zhang, W. Yao, and K. Hashimoto, "BDNF-TrkB signaling in the nucleus accumbens shell of mice has key role in methamphetamine withdrawal symptoms," Translational Psychiatry, vol. 5, no. 10, article no. e666, 2015.

[57] J. Goggi, I. A. Pullar, S. L. Carney, and H. F. Bradford, "The control of [125I]BDNF release from striatal rat brain slices," Brain Research, vol. 967, no. 1-2, pp. 201-209, 2003.

[58] E. M. Anderson, A. M. Wissman, J. Chemplanikal et al., "BDNFTrkB controls cocaine-induced dendritic spines in rodent nucleus accumbens dissociated from increases in addictive behaviors," Proceedings of the National Acadamy of Sciences of the United States of America, vol. 114, no. 35, pp. 9469-9474, 2017.

[59] D.-M. Duan, Y. Tu, P. Liu, and S. Jiao, "Antidepressant effect of electroacupuncture regulates signal targeting in the brain and increases brainderived neurotrophic factor levels," Neural Regeneration Research, vol. 11, no. 10, pp. 1595-1602, 2016.

[60] X. Ji, Q. Wang, and W. Zhu, "Expressions of neurotransmitters in patients of insomnia differentiated as liver stagnation transforming into fire treated with acupuncture," Zhongguo Zhen Jiu, vol. 35, no. 6, pp. 549-552, 2015.

[61] J.-L. Yang, R. Zhang, L. Du, Y.-S. Yang, and X.-C. Liu, "Clinical observation on the neurotransmitters regulation in patients of insomnia differentiated as yang deficiency pattern treated with warm acupuncture and auricular point sticking therapy," Zhongguo Zhen Jiu, vol. 34, no. 12, pp. 1165-1168, 2014.

[62] H. Malinen and P. Hyytiä, "Ethanol self-administration is regulated by $\mathrm{CB} 1$ receptors in the nucleus accumbens and ventral tegmental area in alcohol-preferring AA rats," Alcoholism: Clinical and Experimental Research, vol. 32, no. 11, pp. 19761983, 2008

[63] J. C. Escosteguy-Neto, P. Fallopa, P. Varela, R. Filev, A. Tabosa, and J. G. Santos-Junior, "Electroacupuncture inhibits CB1 
upregulation induced by ethanol withdrawal in mice," Neurochemistry International, vol. 61, no. 2, pp. 277-285, 2012.

[64] B. Sperlágh, K. Windisch, R. D. Andó, and E. Sylvester Vizi, "Neurochemical evidence that stimulation of CB1 cannabinoid receptors on GABAergic nerve terminals activates the dopaminergic reward system by increasing dopamine release in the rat nucleus accumbens," Neurochemistry International, vol. 54, no. 7, pp. 452-457, 2009.

[65] A. Cippitelli, A. Bilbao, A. C. Hansson et al., "Cannabinoid $\mathrm{CB} 1$ receptor antagonism reduces conditioned reinstatement of ethanol-seeking behavior in rats," European Journal of Neuroscience, vol. 21, no. 8, pp. 2243-2251, 2005.

[66] T. J. De Vries, Y. Shaham, J. R. Homberg et al., "A cannabinoid mechanism in relapse to cocaine seeking," Nature Medicine, vol. 7, no. 10, pp. 1151-1154, 2001.

[67] L. G. Schmidt, J. Samochowiec, U. Finckh et al., "Association of a CB1 Cannabinoid Receptor Gene (CNR1) polymorphism with severe alcohol dependence," Drug and Alcohol Dependence, vol. 65, no. 3, pp. 221-224, 2002.

[68] C. H. Yang, S. S. Yoon, D. M. Hansen et al., "Acupuncture inhibits GABA neuron activity in the ventral tegmental area and reduces ethanol self-administration," Alcoholism: Clinical and Experimental Research, vol. 34, no. 12, pp. 2137-2146, 2010.

[69] Q. Wang, L.-M. Mao, Y.-S. Shi, and J.-S. Han, "Lumbar intrathecal administration of naloxone antagonizes analgesia produced by electrical stimulation of the hypothalamic arcuate nucleus in pentobarbital-anesthetized rats," Neuropharmacology, vol. 29, no. 12, pp. 1123-1129, 1990.

[70] Q. Wang, L. Mao, and J. Han, "Characterization of inhibition of spinal nociceptive reflex by stimulation of the arcuate nucleus of the hypothalamus in the pentobarbital-anesthetized rat," PAIN, vol. 41, no. 1, pp. 101-108, 1990.

[71] H. L. Wen, W. K. K. Ho, L. Ma, G. H. Choa, and N. Ling, "The influence of electro-acupuncture on naloxone-induced morphine withdrawal: II. Elevation of immunoassayable betaendorphin activity in the brain but not the blood," American Journal of Chinese Medicine, vol. 7, no. 3, pp. 237-240, 1979.

[72] A. Mansour, H. Khachaturian, M. E. Lewis, H. Akil, and S. J. Watson, "Anatomy of CNS opioid receptors," Trends in Neurosciences, vol. 11, no. 7, pp. 308-314, 1988.

[73] C. H. Yang, B. H. Lee, and S. H. Sohn, "A possible mechanism underlying the effectiveness of acupuncture in the treatment of drug addiction," Evidence-Based Complementary and Alternative Medicine, vol. 5, no. 3, Article ID 463284, 266 pages, 2008.

[74] A. Zangen and U. Shalev, "Nucleus accumbens $\beta$-endorphin levels are not elevated by brain stimulation reward but do increase with extinction," European Journal of Neuroscience, vol. 17, no. 5, pp. 1067-1072, 2003.

[75] O. Nakagawasai, T. Tadano, and K. Tan No, "Changes in betaendorphin and stress-induced analgesia in mice after exposure to forced walking stress," Methods and Findings in Experimental and Clinical Pharmacology, vol. 21, no. 7, pp. 471-476, 1999.

[76] J. Liang, X. J. Ping, Y. J. Li et al., "Morphine-induced conditioned place preference in rats is inhibited by electroacupuncture at 2 Hz: Role of enkephalin in the nucleus accumbens," Neuropharmacology, vol. 58, no. 1, pp. 233-240, 2010.

[77] K. Sakatani, M. Fujii, N. Takemura, and T. Hirayama, "Effects of acupuncture on anxiety levels and prefrontal cortex activity measured by near-infrared spectroscopy: A Pilot Study," Advances in Experimental Medicine and Biology, vol. 876, pp. 297-302, 2016.
[78] M. Dias, D. Pagnin, V. De Queiroz Pagnin, R. L. R. Reis, and B. Olej, "Effects of electroacupuncture on stress-related symptoms in medical students: A randomised controlled pilot study," Acupuncture in Medicine, vol. 30, no. 2, pp. 89-95, 2012.

[79] H.-J. Park, Y. Chae, J. Jang, I. Shim, H. Lee, and S. Lim, "The effect of acupuncture on anxiety and neuropeptide $\mathrm{Y}$ expression in the basolateral amygdala of maternally separated rats," Neuroscience Letters, vol. 377, no. 3, pp. 179-184, 2005.

[80] Y. Zhao, C. Cui, X. Yu et al., "Electroacupuncture ameliorates abnormal defaecation and regulates corticotrophin-releasing factor in a rat model of stress," Acupuncture in Medicine, vol. 35, no. 2, pp. 114-121, 2017.

[81] Z. Zhao, B. H. Lee, F. Lin et al., "Effects of acupuncture at $\mathrm{Zu}-\mathrm{San}-\mathrm{Li}$ (ST36) on the activity of the hypothalamicpituitary-adrenal axis during ethanol withdrawal in rats," Journal of Acupuncture and Meridian Studies, vol. 7, no. 5, pp. 225-230, 2014.

[82] O. George, S. Ghozland, M. R. Azar et al., "CRF-CRF1 system activation mediates withdrawal-induced increases in nicotine self-administration in nicotine-dependent rats," Proceedings of the National Acadamy of Sciences of the United States of America, vol. 104, no. 43, pp. 17198-17203, 2007.

[83] M. F. Olive, H. N. Koenig, M. A. Nannini, and C. W. Hodge, "Elevated extracellular CRF levels in the bed nucleus of the stria terminalis during ethanol withdrawal and reduction by subsequent ethanol intake," Pharmacology Biochemistry \& Behavior, vol. 72, no. 1-2, pp. 213-220, 2002.

[84] J. D. Shepard, K. W. Barron, and D. A. Myers, "Corticosterone delivery to the amygdala increases corticotropin-releasing factor mRNA in the central amygdaloid nucleus and anxiety-like behavior," Brain Research, vol. 861, no. 2, pp. 288-295, 2000.

[85] C. K. Funk, L. E. O’Dell, E. F. Crawford, and G. F. Koob, "Corticotropin-releasing factor within the central nucleus of the amygdala mediates enhanced ethanol self-administration in withdrawn, ethanol-dependent rats," The Journal of Neuroscience, vol. 26, no. 44, pp. 11324-11332, 2006.

[86] S. Liu, W. Zhou, H. Liu, G. Yang, and W. Zhao, "Electroacupuncture attenuates morphine withdrawal signs and cFos expression in the central nucleus of the amygdala in freely moving rats," Brain Research, vol. 1044, no. 2, pp. 155-163, 2005.

[87] L. Eshkevari, E. Permaul, and S. E. Mulroney, "Acupuncture blocks cold stress-induced increases in the hypothalamuspituitary-adrenal axis in the rat," Journal of Endocrinology, vol. 217, no. 1, pp. 95-104, 2013.

[88] Z. Zhao, X. Jin, Y. Wu et al., "Amygdaloid corticotropinreleasing factor is involved in the anxiolytic effect of acupuncture during ethanol withdrawal in rats," Journal of Acupuncture and Meridian Studies, vol. 6, no. 5, pp. 234-240, 2013.

[89] Y. Chae, M. Yeom, J.-H. Han et al., "Effect of acupuncture on anxiety-like behavior during nicotine withdrawal and relevant mechanisms," Neuroscience Letters, vol. 430, no. 2, pp. 98-102, 2008.

[90] Z. Zhao, S. C. Kim, Y. Wu et al., "Involvement of amygdaloid neuropeptide $\mathrm{Y}$ in the anxiolytic effects of acupuncture during ethanol withdrawal in rats," Neuroscience Letters, vol. 567, pp. 19-23, 2014.

[91] L. T. Zhu and Z. Z. Tian, "Effect of electroacupuncture intervention on expression of corticotropin-releasing factor protein in hypothalamus and hippocampus of traumatized rats," Zhen Ci Yan Jiu, vol. 38, no. 5, pp. 369-374, 2013.

[92] N. I. Boyadjieva, M. Ortigüela, A. Arjona, X. Cheng, and D. K. Sarkar, " $\beta$-endorphin neuronal cell transplant reduces 
corticotropin releasing hormone hyperresponse to lipopolysaccharide and eliminates natural killer cell functional deficiencies in fetal alcohol exposed rats," Alcoholism: Clinical and Experimental Research, vol. 33, no. 5, pp. 931-937, 2009.

[93] R. W. Logan, O. Wynne, G. Maglakelidze et al., “ $\beta$-Endorphin Neuronal Transplantation Into the Hypothalamus Alters Anxiety-Like Behaviors in Prenatal Alcohol-Exposed Rats and Alcohol-Non-Preferring and Alcohol-Preferring Rats," Alcoholism: Clinical and Experimental Research, vol. 39, no. 1, pp. 146-157, 2015.

[94] A. R. Childress, P. D. Mozley, W. McElgin, J. Fitzgerald, M. Reivich, and C. P. O'Brien, "Limbic activation during cueinduced cocaine craving," The American Journal of Psychiatry, vol. 156, no. 1, pp. 11-18, 1999.

[95] J. R. Mantsch, D. A. Baker, D. Funk, A. D. Lê, and Y. Shaham, "Stress-induced reinstatement of drug seeking: 20 years of progress," Neuropsychopharmacology, vol. 41, no. 1, pp. 335-356, 2016.

[96] P. W. Czoty, "Lack of effect of ethanol on cocaine prime-induced reinstatement of extinguished cocaine self-administration in rhesus monkeys," Behavioural Pharmacology, vol. 27, no. 7, pp. 633-636, 2016.

[97] P. E. Phillips, G. D. Stuber, M. L. Heien, R. M. Wightman, and R. M. Carelli, "Subsecond dopamine release promotes cocaine seeking," Nature, vol. 422, no. 6932, pp. 614-618, 2003.

[98] U. Shalev, J. W. Grimm, and Y. Shaham, "Neurobiology of relapse to heroin and cocaine seeking: A review," Pharmacological Reviews, vol. 54, no. 1, pp. 1-42, 2002.

[99] C. Lowman, J. Allen, R. L. Stout, and T. R. Group, "Replication and extension of Marlatt's taxonomy of relapse precipitants: overview of procedures and results," Addiction, vol. 91, no. 12, pp. 51-71, 1996.

[100] E. P. Zorrilla, M. L. Logrip, and G. F. Koob, "Corticotropin releasing factor: A key role in the neurobiology of addiction," Frontiers in Neuroendocrinology, vol. 35, no. 2, pp. 234-244, 2014.

[101] S. A. Kim, B. H. Lee, J. H. Bae et al., "Peripheral afferent mechanisms underlying acupuncture inhibition of cocaine behavioral effects in rats," PLoS ONE, vol. 8, no. 11, Article ID e81018, 2013.

[102] J. S. Han, "Acupuncture: neuropeptide release produced by electrical stimulation of different frequencies," Trends in Neurosciences, vol. 26, no. 1, pp. 17-22, 2003.

[103] S. S. Yoon, E. J. Yang, B. H. Lee et al., "Effects of acupuncture on stress-induced relapse to cocaine-seeking in rats," Psychopharmacology, vol. 222, no. 2, pp. 303-311, 2012.

[104] M. A. Klitenick, P. DeWitte, and P. W. Kalivas, "Regulation of somatodendritic dopamine release in the ventral tegmental area by opioids and GABA: An in vivo microdialysis study," The Journal of Neuroscience, vol. 12, no. 7, pp. 2623-2632, 1992.

[105] R. van Zessen, J. L. Phillips, E. A. Budygin, and G. D. Stuber, "Activation of VTA GABA Neurons Disrupts Reward Consumption," Neuron, vol. 73, no. 6, pp. 1184-1194, 2012.

[106] W. Jin, M. S. Kim, E. Y. Jang et al., "Acupuncture reduces relapse to cocaine-seeking behavior via activation of GABA neurons in the ventral tegmental area," Addiction Biology, vol. 23, no. 1, pp. 165-181, 2018.

[107] Y. H. Wang, L. B. Zeng, Y. J. Liu et al., "Effect of electroacupuncture on drug-seeking behaviors induced by heroin priming and FosB expression in relevant brain regions," Zhong Nan Da Xue Xue Bao Yi Xue Ban, vol. 33, no. 4, pp. 299-304, 2008.
[108] X. Cai, X. Song, C. Li, C. Xu, X. Li, and Q. Lu, "Acupuncture inhibits cue-induced heroin craving and brain activation," Neural Regeneration Research, vol. 7, no. 33, pp. 2607-2616, 2012.

[109] B. H. Lee, J. H. Ma, S. In et al., "Acupuncture at SI5 attenuates morphine seeking behavior after extinction," Neuroscience Letters, vol. 529, no. 1, pp. 23-27, 2012.

[110] B. H. Lee, S. C. Lim, H. J. Jeon et al., "Acupuncture suppresses reinstatement of morphine-seeking behavior induced by a complex cue in rats," Neuroscience Letters, vol. 548, pp. 126-131, 2013.

[111] M. R. Kim, J. K. Soo, S. L. Yeoung et al., "Effect of acupuncture on behavioral hyperactivity and dopamine release in the nucleus accumbens in rats sensitized to morphine," Neuroscience Letters, vol. 387, no. 1, pp. 17-21, 2005.

[112] B. Lee, S.-M. Han, and I. Shim, "Acupuncture attenuates cocaine-induced expression of behavioral sensitization in rats: possible involvement of the dopaminergic system in the ventral tegmental area," Neuroscience Letters, vol. 449, no. 2, pp. 128132, 2009.

[113] C.-L. Cui, L.-Z. Wu, and F. Luo, "Acupuncture for the treatment of drug addiction," Neurochemical Research, vol. 33, no. 10, pp. 2013-2022, 2008.

[114] C. Southern, C. Lloyd, J. Liu et al., "Acupuncture as an intervention to reduce alcohol dependency: A systematic review and meta-analysis," Chinese Medicine, vol. 11, no. 1, article no. 49, 2016.

[115] J. Fang, D. Ma, J. Zhong et al., "Prospective study of Han's acupoint nerve stimulator for preventing relapse of detoxified heroin addicts," Zhonghua Yi Xue Za Zhi, vol. 90, no. 21, pp. 1472-1476, 2010.

[116] S. Y. Kang, O. S. Kwon, J.-Y. Moon et al., "Mechanical stimulation of the HT7 acupuncture point to reduce ethanol selfadministration in rats," Evidence-Based Complementary and Alternative Medicine, vol. 2017, Article ID 6578621, 6 pages, 2017.

[117] J. Li, Y. Zou, and J.-H. Ye, "Low frequency electroacupuncture selectively decreases voluntarily ethanol intake in rats," Brain Research Bulletin, vol. 86, no. 5-6, pp. 428-434, 2011.

[118] J. S. Levin, T. A. Glass, L. H. Kushi, J. R. Schuck, L. Steele, and W. B. Jonas, "Quantitative Methods in Research on Complementary and Alternative Medicine: A Methodological Manifesto," Medical Care, vol. 35, no. 11, pp. 1079-1094, 1997.

[119] C. H. Yang, S. H. Choi, and J. S. Kim, "The Effects of Acupuncture Stimulation for Brain Activation and Alcohol Abstinence Self-Efficacy: Functional MRI Study," Evidence-Based Complementary and Alternative Medicine, vol. 2017, Article ID 2850124, 7 pages, 2017.

[120] O.-S. Kang, S.-Y. Kim, G.-H. Jahng et al., "Neural substrates of acupuncture in the modulation of cravings induced by smoking-related visual cues: an FMRI study," Psychopharmacology, vol. 228, no. 1, pp. 119-127, 2013.

[121] J.-M. Mu, L. Liu, W. Fang, J.-M. Cheng, and L. Zhao, "Effect of electroacupuncture at Jiaji (EX-B 2) on drug craving of heroin addicts and beta-EP and Dyn-A in plasma," Zhongguo Zhen Jiu, vol. 30, no. 11, pp. 881-885, 2010.

[122] J. M. Wu, D. Y. Wei, Y. F. Luo et al., "Clinic reaseach on heroin de-addiction effects of acupuncture and its potentiality of preventing relapse," Zhong Xi Yi Jie He Xue Bao, vol. 1, no. 4, pp. 268-272, 2003.

[123] L. Lu, Y. Liu, W. Zhu, J. Shi, W. Ling, and T. R. Kosten, "Traditional medicine in the treatment of drug addiction," The American Journal of Drug and Alcohol Abuse, vol. 35, no. 1, pp. 1-11, 2009. 
[124] X. Wang, Z. Wang, J. Liu et al., "Repeated acupuncture treatments modulate amygdala resting state functional connectivity of depressive patients," NeuroImage: Clinical, vol. 12, pp. 746752, 2016.

[125] Z. Wang, X. Wang, J. Liu et al., "Acupuncture treatment modulates the corticostriatal reward circuitry in major depressive disorder," Journal of Psychiatric Research, vol. 84, pp. 18-26, 2017. 


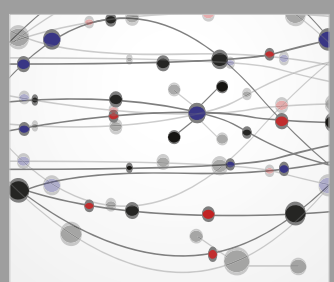

The Scientific World Journal
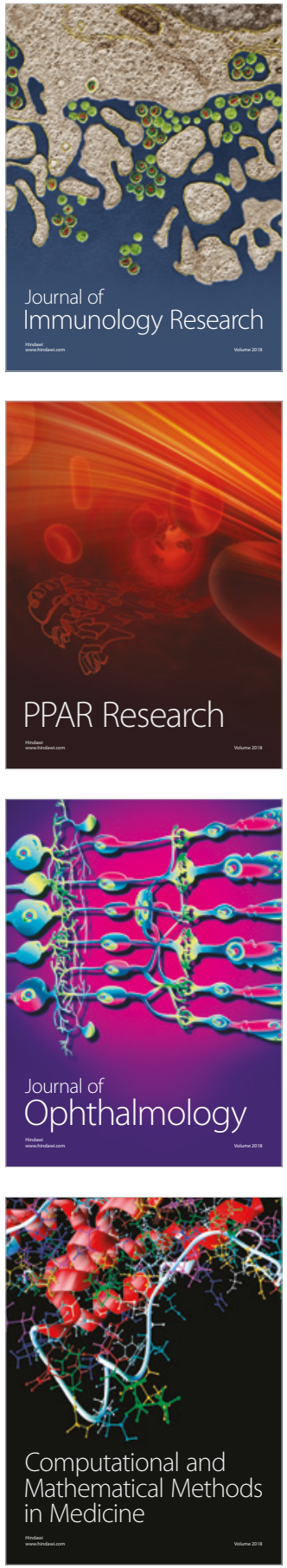

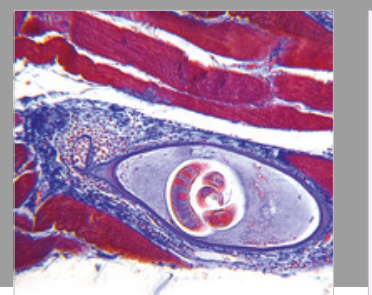

Gastroenterology Research and Practice

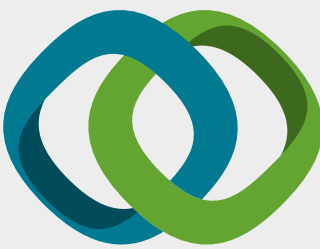

\section{Hindawi}

Submit your manuscripts at

www.hindawi.com
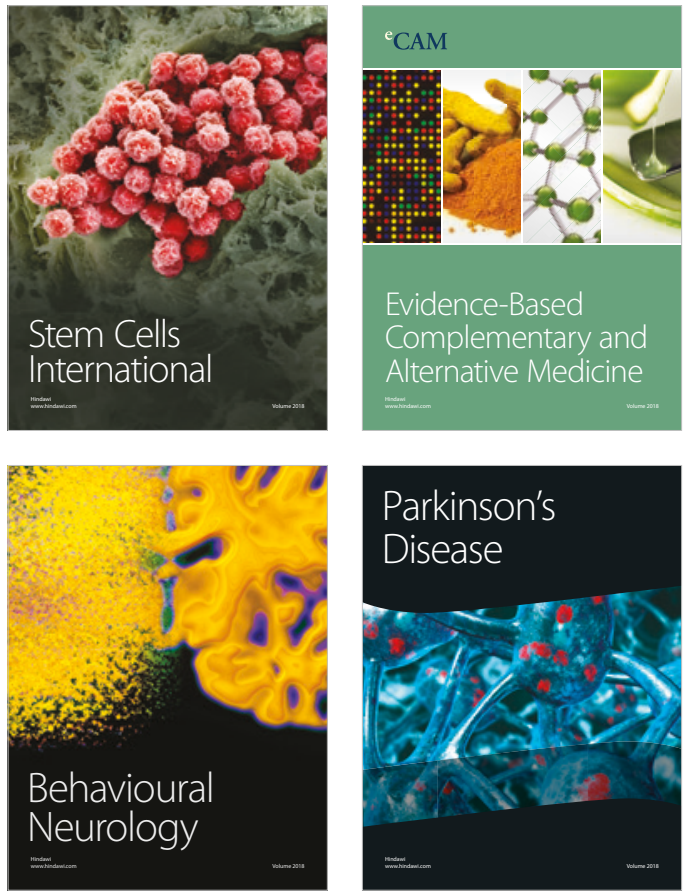

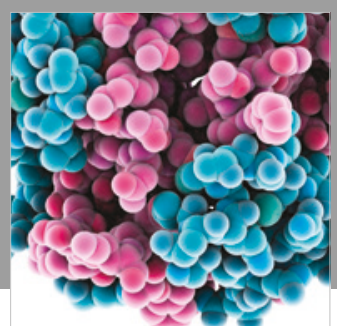

ournal of

Diabetes Research

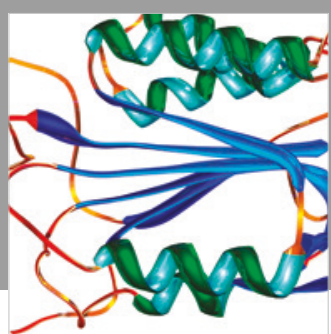

Disease Markers
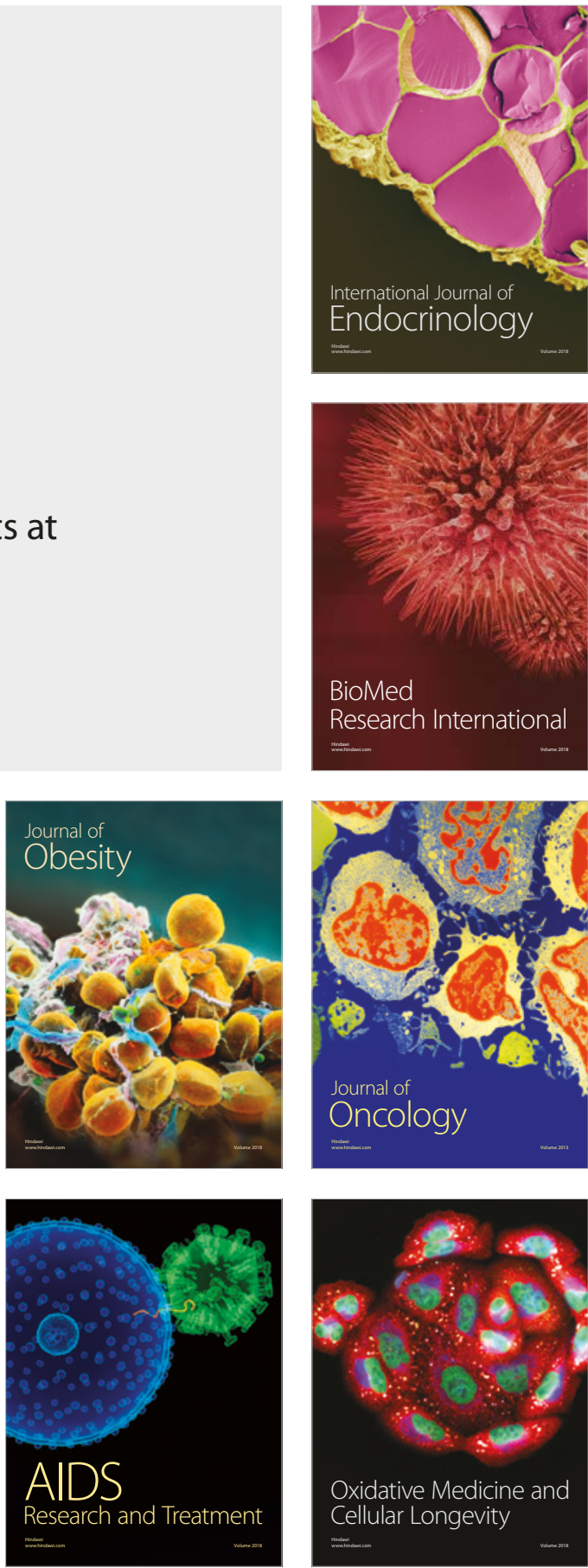